\title{
Anterolateral structure (ALS) injury is significant risk factor for grade 3 pivot shift in knees with acute ACL injuries
}

Andrea Ferretti

Edoardo Monaco

Edoardo Gaj

Valerio Andreozzi

Alessandro Annibaldi

Alessandro Carrozzo

Thais Dutra Vieira

Bertrand Sonnery-Cottet

Adnan Saithna

\section{Video Abstract}

Keywords: The American Journal of Sports Medicine, ACL, ALS, pivot shift, tibial slope, anterior cruciate ligament, anterolateral structure, instability, ACL reconstruction, MRI, International Knee Documentation Committee, IKDC, soft tissue, osseous factors, knee laxity, pivot shift test, medial collateral ligament, MCL, anesthesia, meniscus

Posted Date: October 23rd, 2020

DOI: https://doi.org/10.21203/rs.3.rs-97529/v1

License: (1) (1) This work is licensed under a Creative Commons Attribution 4.0 International License. Read Full License 


\section{Abstract}

Among patients undergoing ACL reconstruction surgery, a high-grade pivot shift before surgery is widely considered to confer an increased risk of graft rupture and persistent instability after surgery. But what are the risk factors for high-grade pivot shifts themselves? A new study published in The American Journal of Sports Medicine reports injury to anterolateral structures as the most important risk factor for high-grade pivot shifts in patients suffering ACL injuries. The pivot shift test is the most specific physical exam for diagnosing ACL injuries. The examiner applies inward pressure on the lateral thigh with one hand while internally rotating the lower leg as the knee is flexed from a fully extended position. The presence of a pivot shift is indicated by an abrupt forward shift of the tibia back to its normal anatomical position. This shift is graded between 1 and 3 according International Knee Documentation Committee criteria, with 1 and 2 considered low grade, and 3 considered high grade. To understand the circumstances that give rise to such pivot shifts, researchers examined the knees of 200 patients undergoing ACL reconstruction within 10 days of injury: 35 had a grade 3 pivot shift, whereas 165 had a grade 1 or 2 pivot shift. The researchers performed a comprehensive evaluation of soft tissue and bone parameters. Soft tissue injuries included tears of the meniscus and/or MCL; lesions of anterolateral structures, or ALS; and chondral injuries. Bone-related parameters such as tibial slope and condylar ratios were determined using established MRI procedures. A multivariate analysis revealed that ALS injury was the only significant risk factor associated with a grade 3 pivot shift. The finding is consistent with previous studies linking abnormalities of the ALS to a high probability of experiencing a high-grade pivot shift among patients undergoing ACL reconstruction. More work is needed to clarify the connection between ALS injury and pivot shift. Future studies could, for example, use a more quantitative measure of the extent of pivot shift, such as tibial acceleration. And they could explore a larger sample of patient knees to better understand the range of factors that lead to re-injury among patients undergoing ACL reconstruction surgery. 